\title{
Emission Spectrum of Carbon Monoxide From 2.3 to 2.5 Microns
}

\author{
Earle K. Plyler, Harry C. Allen, Jr., and E. D. Tidwell
}

\begin{abstract}
The 2-0, 3-1, 4-2, 5-3, and 6-4 emission bands of carbon monoxide have been measured in the region from 4000 to $4360 \mathrm{~cm}^{-1}$ with a grating spectrometer. A 10,000 line per inch grating used in the second order resolved lines separated by $0.08 \mathrm{~cm}^{-1}$. Several sets of measurements were made on the $2-0$ band and the molecular constants were calculated. The constants in $\mathrm{cm}^{-1}$ are $B_{0}=1.922511 \pm 0.000025 \quad D_{0}=6.13 \pm 0.02 \times 10^{-6}$. Using these values and the $B_{0}$ from $1-0$ band in conjunction with the microwave constant a new determination of the speed of light is obtained, $c=299,794 \pm 3$ kilometers per second.
\end{abstract}

\section{Introduction}

The vibrational-rotational spectrum of carbon monoxide in emission was first measured in the infrared region in $1951 \mid 1] .^{2}$ The observations were made on the harmonic band in the region from 4000 to $4360 \mathrm{~cm}^{-1}$. Due to the high temperature of the flame the $2-0,3-1,4-2$, and $5-3$ transitions were observed. Recently Goldberg and Muller [2] have measured the absorption spectrum of carbon monoxide in the solar spectrum and have observed rotational lines with $J$ values up to 75 .

Other bands of carbon monoxide have also been measured. Herzberg and Rao [3] have measured the $4-0$ band of $\mathrm{CO}$ in absorption in the near infrared, and the fundamental band at $4.67 \mu$ has been measured by Plyler, Blaine, and Tidwell [4]. More recently 33 lines of the 2-0 band of $\mathrm{CO}$ have been measured by Rank and his co-workers [5]. From each set of measurements the various workers have calculated a set of molecular constants for the $\mathrm{CO}$ molecule and the results of different authors $[2,4,5]$ are in close agreement.

Since the earlier measurements were made on the harmonic band of $\mathrm{CO}$, improvements in detectors and infrared spectrometers permit more accurate measurements to be made. The bands in the 4000 to $4360 \mathrm{~cm}^{-1}$ region have been remeasured and this paper gives the results which have been obtained.

\section{Experimental Method and Results}

The emission from carbon monoxide in the $2.3-\mu$ region was produced in two ways. First, a tube containing $\mathrm{CO}$ was placed in the coil of a hf oscillator. (The use of a hf oscillator for the production of molecular spectra has been previously studied by Wilkinson, Ford, and Price [6].) The pressure of the $\mathrm{CO}$ was $6 \mathrm{~cm}$ and a small flow of fresh gas was maintained. A spectrum of high intensity was observed showing transitions up to 11-9. The intensity of the rotational lines, however, varied with the pressure and the current through the oscillator coil and it was felt that more precise measurements of the

1 This work was supported by the Geophysics Research Directorate, Air Force. Cambridge Research Center.

${ }^{2}$ Figures in brackets indicate the literature references at the end of this paper. lines could be made of the spectrum from a flame. Second, the CO spectrum was observed in emission from an oxyacetylene flame. The gases were premixed and were slightly rich in fuel. The burning took place in a welding torch and a section of the flame about 2 in. above the inner cone was focused on the front slit of the spectrometer. The radiation falling on the slit was increased by reflections of mirrors around the flame [7]. A grating spectrometer with a collimating mirror of one-meter focal length and a grating of 10,000 lines/in. was used for resolving the bands. The spectrum was observed in the first and second orders, the second order giving higher resolution. Emission lines separated by 0.08 $\mathrm{cm}^{-1}$ were partially resolved.

The observed spectrum (4000 to $\left.4360 \mathrm{~cm}^{-1}\right)$ includes the transitions $2-0,3-1,4-2,5-3,6-4$, and $7-5$. Because of the overlapping of the lines from the different transitions, precise measurements were made on the bands arising from the three lowest transitions. In figure 1 are shown the band heads and some of the rotational lines for the $2-0,3-1,4-2$, 5-3, and 6-4 transitions. The intensity of the rotational lines with the same $J$ values of the $2-0$ and the $3-1$ is about equal. In the other transitions the intensity is lower. In order to obtain sizeable deflections it was necessary to increase the amplifier gain for the other transitions; the noise level was the greatest for the 6-4 band.

The small lines appearing between the strong lines from R30 to R45 in each band arise from the band folding back on itself; these lines are part of the $R$ branch extending up to about $J=68$. The high $J$ values are almost superimposed on the lower $J$ 's in the 2-0 transition, but in the $3-1,4-2$, and $5-3$ transitions the high $J$ lines are well separated from the stronger lines in the region $\mathrm{R} 30$ to $\mathrm{R} 45$.

Some of the low-intensity lines observed in the 3-1 region are the $\mathrm{R} 3$ to $\mathrm{R} 6$ lines of the $2-0$ band. Their low intensity shows that very few of the molecules in the flame exist in the lower rotational states. On figure 1 are given the identifications of the different lines. Precise measurements were made on the $2-0,3-1$, and $4-2$ bands. When the lines of the different series overlapped they were not measured. In order to have a more complete set of measurements of the 2-0 band the lines from R20 
to P20 were measured in absorption. The calculated and observed wave numbers of the $2-0,3-1$, and 4-2 bands are given in table 1.

\section{Discussion of Results}

The molecular constants of $\mathrm{CO}$ have been calculated from measurements of the $4-0,2-0$, and 1-0 bands by several observers $[1,2,3,4,5]$. In most

cases the constants determined by Goldberg and Muller; Plyler, Blaine, and Tidwell; and Rank agree within the probable error given by the authors.

The line positions measured in the $2-0$ band have been used to calculate $B_{0}, D_{0}$ and $\nu_{0}$ by the equation,

$$
\begin{aligned}
\nu=\nu_{0}+\left(B_{2}+B_{0}\right) m+ & \left(B_{2}-B_{0}-D_{2}+D_{0}\right) m^{2}- \\
& 2\left(D_{2}+D_{0}\right) m^{3}-\left(D_{2}-D_{0}\right) m^{4} .
\end{aligned}
$$

TABLE 1. Observed frequencies $\left(\mathrm{cm}^{-1}\right)$ and deviations from calculated values of $\mathrm{CO}$ lines from emission measurements

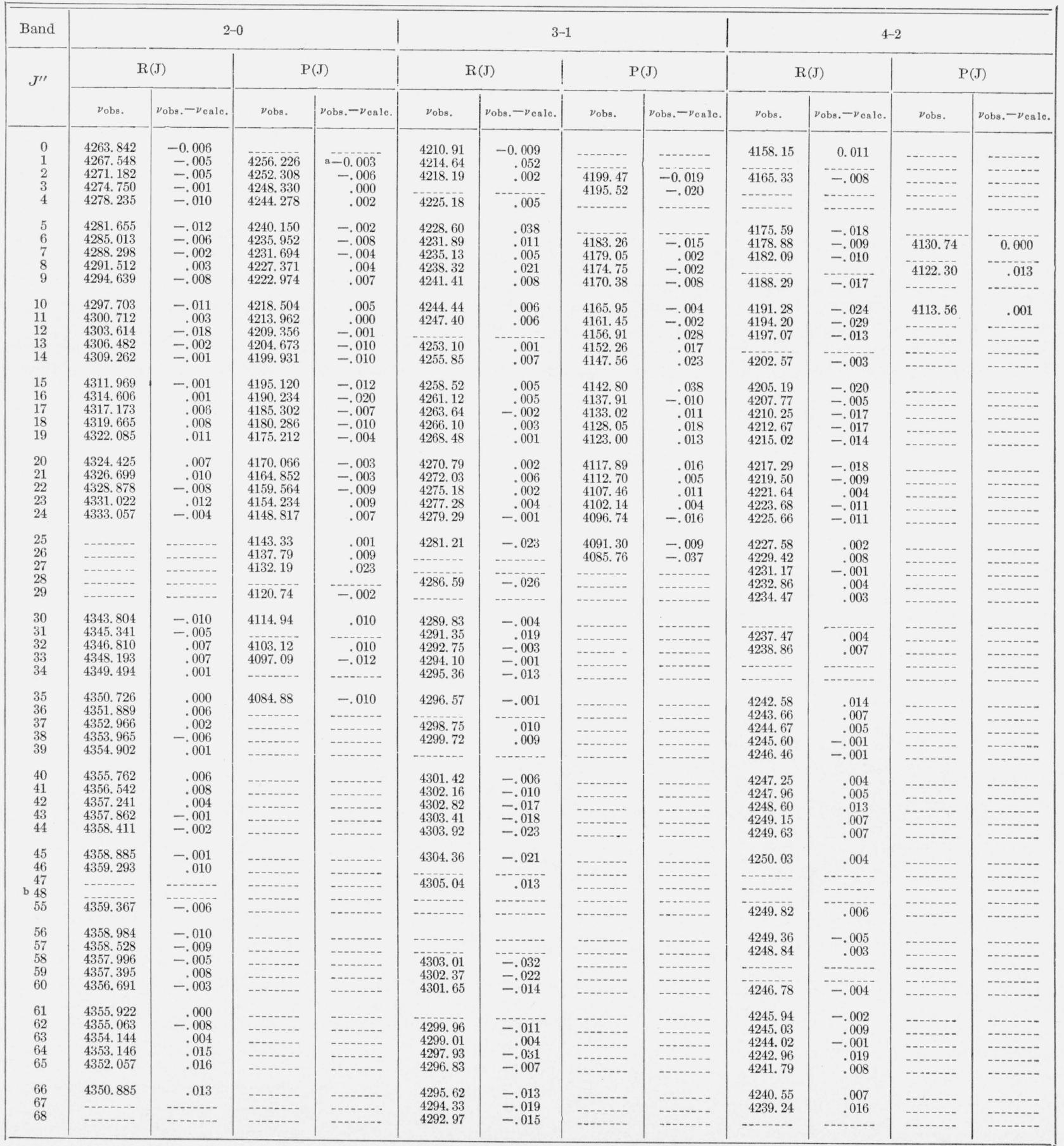

a Lines from R20 to P20 measured in absorption. b No measurements were taken for $J^{\prime \prime}=48$ through 54 . 


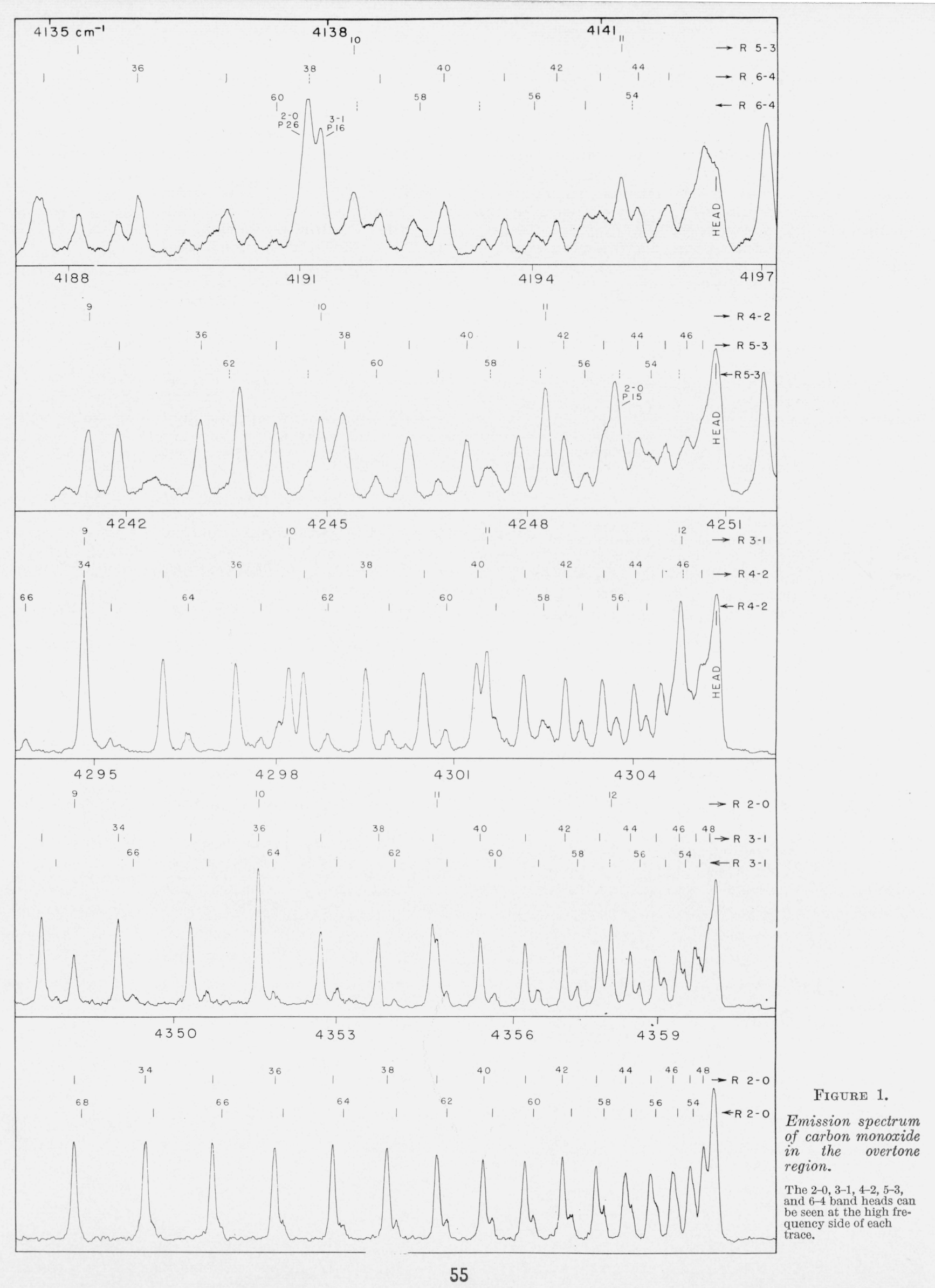


The constants were calculated both with and without the $m^{4}$ term. Only very small differences occurred in $\nu_{0}, B_{0}$, and $D_{0}$ when the fourth power term was omitted. Since $D_{2}-D_{0}$ is about $2 \times 10^{-9} \mathrm{~cm}^{-1}$, the contribution of this term for $J=20$ is approximately $0.001 \mathrm{~cm}^{-1}$ which is about one-fourth the experimental error in the measurements of the lines. Sixty-five lines of the 2-0 band were used in the calculation. They extended from R46 to P24 (see table 1). The constants obtained, in $\mathrm{cm}^{-1}$, were

$$
\begin{aligned}
\nu_{0} & =4260.069 \pm 0.001 \\
B_{0} & =1.922511 \pm 0.000028 \\
D_{0} & =6.13 \pm 0.022 \times 10^{-6} .
\end{aligned}
$$

The constants $B_{0}$ and $D_{0}$ had been previously calculated from measurements of the $1-0$ band [8] and found to be

$$
\begin{aligned}
& B_{0}=1.922523 \pm 0.000025 \\
& D_{0}=6.26 \pm 0.17 \times 10^{-6} .
\end{aligned}
$$

The standard deviations were first reported but the uncertainty is now given as probable error. The values of $B_{0}$ from the two bands fall within the error of measurements. A better value of $B_{0}$ can be obtained by combining [8] the two $B_{0}$ 's determined by analyses of the 1-0 and 2-0 bands, which gives the result,

$$
B_{0}=1.922517 \pm 0.000019
$$

the uncertainty again being the probable error.

The central value of $D_{0}$ as determined from an analysis of the 2-0 band is in better agreement with the recent value of $D_{0}=6.131 \times 10^{-6}$ obtained by Gordy from the microwave spectrum [9], and the value of $D_{0}=6.117 \times 10^{-6}$ from a measurement of the 2-0 by Rank et al. [5] than was the earlier determination from the $1-0$ band.

There are now two determinations for $B_{0}$ which give an average value of $1.922517 \pm 0.000019$. This value of $B_{0}$ is in excellent agreement with the values obtained by Rank et al. [5],

and

$$
B_{0}=1.922519 \pm 0.000013
$$

$$
B_{0}=1.922521 \pm 0.0000035 \text {. }
$$

The first value was obtained from the least squares treatment of $13 \Delta_{2} F^{\prime \prime}$ values from the measurements, and the second value was the result of using a calculated value for the constant $D_{0}$ and including only the 8 largest $\Delta_{2} F^{\prime \prime}$ values in the least squares treatment. Rank et al. [5] have calculated the velocity of light using the second $B_{0}$ given above and from $B_{0}=57,635.965 \pm 0.005 \mathrm{Mc}$ determined from the rotational spectrum. The result is $c=$ $299,793.7 \pm 0.7 \mathrm{~km} / \mathrm{sec}$. On using the same value of $B_{0}$ from microwave measurements and the average value from our infrared measurements, the speed of light is determined as $c=299,794 \pm 3 \mathrm{~km} / \mathrm{sec}$ in excellent agreement with previous determinations by this method. The central value is somewhat higher than the best value given by Bearden and Thomsen [10], 299,792.8 and also that given by Cohen, DuMond et al. [11], 299,793.0; however both of their values are within the probable error of this determination.

In conclusion, the authors thank Carroll Dannemiller for doing the least squares calculations on the Bureau's electronic computer.

\section{References}

[1] Earle K. Plyler, W. S. Benedict, and S. Silverman, J. Chem. Phys. 20, 175 (1952).

[2] Leo Goldberg and Edith A. Muller, Astrophys. J. 118, 397 (1953).

[3] G. Herzberg and K. V. Rao, J. Chem. Phys. 17, 1099 (1949).

[4] Earle K. Plyler, Lamdin R. Blaine, and Eugene D. Tidwell, J. Research NBS 55, 183 (1955) RP2617.

[5] D. H. Rank, A. H. Guenther, G. D. Saksena, J. N. Shearer, and T. A. Wiggins, J. Opt. Soc. Am. 47, 686 (1957).

[6] Wilkinson, Ford, and Price, Molecular spectroscopy (Institute of Petroleum, London, 1955).

[7] Earl K. Plyler and H. J. Kostkowski, J. Opt. Soc. Am. 42, 360 (1952).

[8] Earle K. Plyler, L. R. Blaine, and W. S. Connor, J. Opt. Soc. Am. 45, 102 (1955).

[9] W. Gordy (private communication).

[10] J. A. Bearden and J. S. Thomsen, A survey of atomic constants (Johns Hopkins University, 1956).

[11] E. R. Cohen, J. W. M. DuMond, et al., Rev. Mod. Phys. 27, 363 (1955).

Washington, February 5, 1958. 